\title{
A Case of Massive Verapamil Ingestion with Bradycardia and Severe Shock: Prehospital Care and Future Possibilities
}

\author{
Casey Patrick ${ }^{1 *}$, Christopher Goodrich ${ }^{1}$, Brad Ward $^{1}$, Jordan Anderson ${ }^{1}$ and Robert Dickson ${ }^{1,2}$ \\ ${ }^{1}$ Montgomery County Hospital District EMS Service, USA \\ ${ }^{2}$ Department of Emergency Medicine, Baylor College of Medicine Texas, USA
}

*Corresponding author: Casey Patrick, Montgomery County Hospital District EMS Service, Conroe,1400 South Loop 336 West

Conroe, Texas, USA

\section{ARTICLE INFO}

Received: 慧 February 02, 2020

Published: 慧 February 13, 2020

Citation: Casey Patrick, Christopher Goodrich, Brad Ward, Jordan Anderson, Robert Dickson. A Case of Massive Verapamil Ingestion with Bradycardia and Severe Shock: Prehospital Care and Future Possibilities. Biomed J Sci \& Tech Res 25(4)2020. BJSTR. MS.ID.004226.
ABSTRACT

We report a case of massive verapamil poisoning with severe bradycardia and shock. The patient was treated aggressively in the prehospital setting with multiple doses of calcium chloride, glucagon, transdermal pacing and push-dose epinephrine. Upon arrival to the emergency department, he was started on high dose euglycemic insulin therapy (HIET), intravenous lipid emulsion (ILE) and a transvenous pacemaker was placed. The patient recovered and was transferred to psychiatric care with no deleterious cardiac complications. Our case demonstrates the need for aggressive, early, prehospital interventions by EMS when treating calcium channel blocker poisoning

Keywords: Calcium Channel Blocker; Toxicology; Prehospital; Emergency Medical Service Shock; Calcium channel blocker; EMS; Toxicology; Prehospital

\section{Case}

A suburban, third-service, municipal, advanced life support ambulance was dispatched to a 48-year-old male for possible ingestion. EMS arrived 8 minutes following dispatch call receipt to find the patient lying supine, pale and clammy, bradycardic with profound shock. Family reported that he had taken an unknown amount of verapamil and levetiracetam prior to EMS arrival. The initial blood pressure (BP) was $50 / 37$ with a pulse of 49 , a respiratory rate of 24 and a room air oxygen saturation of $95 \%$.He was responsive and maintaining his airway yet confused with a GCS of 14.Initial 12-lead ECG demonstrated a junctional bradycardia with widened QRS (151ms) at a rate of 48 (Figure 1). Two peripheral intravenous and humeral intraosseous access sites were obtained, and $0.9 \%$ normal saline (NS) boluses were started. One gram of calcium chloride was administered, and transdermal pacing was started with $140 \mathrm{~mA}$ at a rate of 60 . Transport was initiated after a 34-minute scene time. The patient continued to remain hypotensive despite pacemaker capture, increased pulse, and $1000 \mathrm{~mL}$ NS bolus so two additional grams of calcium chloride were administered along with one gram of glucagon and 20 micrograms of push-dose epinephrine IV while enroute to the ED. Total transport time was 28 minutes.

Upon arrival to the ED, his BP was 123/38 and pulse with transdermal pacing was 99 . The emergency physician was present on arrival, and the initial blood glucose was 350mg/dL. Lipid emulsion therapy $(250 \mathrm{ml} 20 \%$ fat emulsion), 150 units of regular insulin bolus and epinephrine drip at $10 \mathrm{mcg} / \mathrm{kg} / \mathrm{min}$ were started along with immediate cardiology consultation. The patient remained hypotensive (SBP 60-80) and became more altered, so intubation for airway protection was performed. Poison control was consulted, and cardiology evaluated the patient in the ED. A transvenous pacemaker was placed due to pacemaker dependence, continued bradycardia and shock (Figure 2). High dose regular insulin therapy, at a dose of $250 \mathrm{U} / \mathrm{hr}$, was continued over the next 48 hours and titrated as the patient stabilized (Figure 3). His levetiracetam level returned at $299 \mathrm{mcg} / \mathrm{mL}$ which is markedly elevated and could've contributed to the initial depressed mental 
status [1]. Steady improvement was noted during the hospital stay and support was weaned. On hospital day 6, the patient was awake, alert, hemodynamically stable and able to be transferred for further psychiatric treatment.

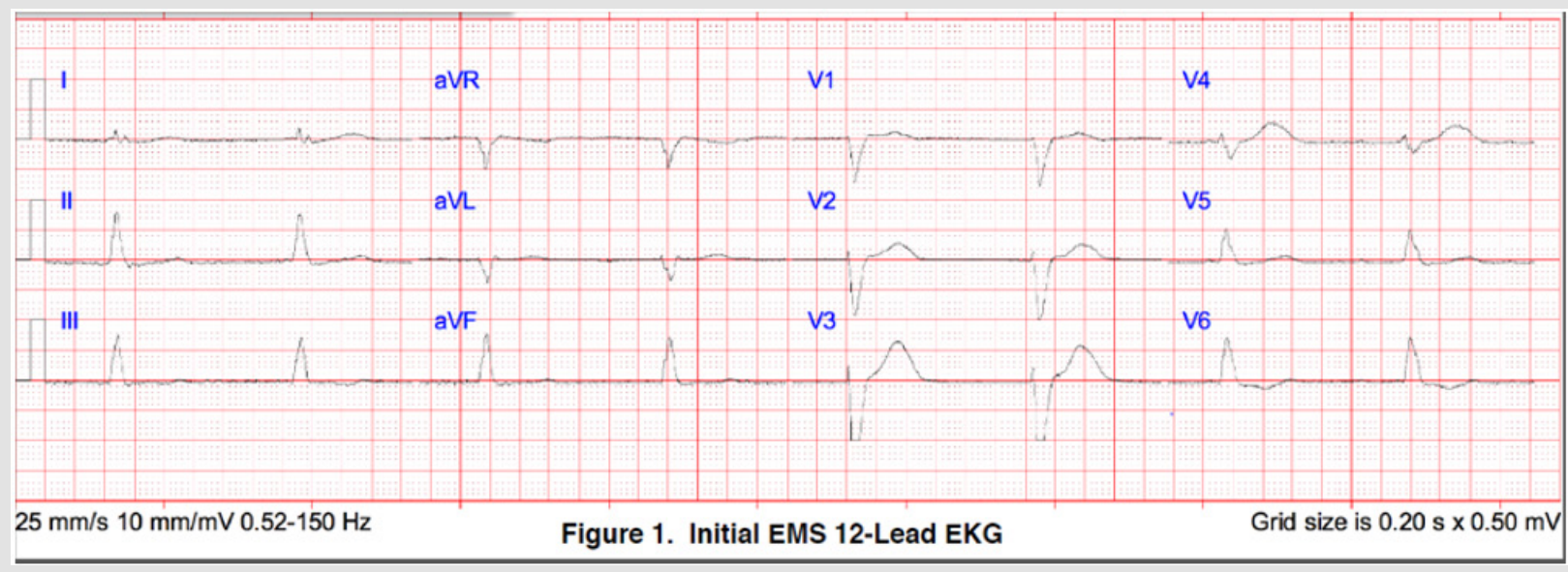

Figure 1: Initial EMS 12-Lead EKG.

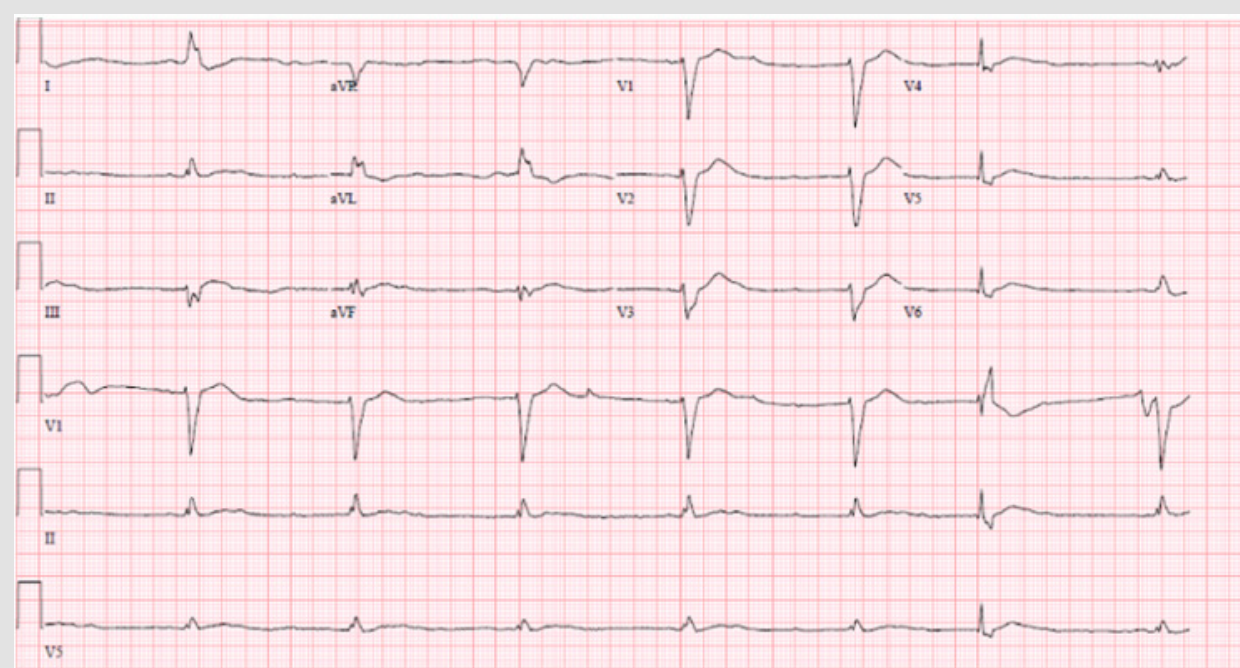

Figure 2: Initial Emergency Department EKG.

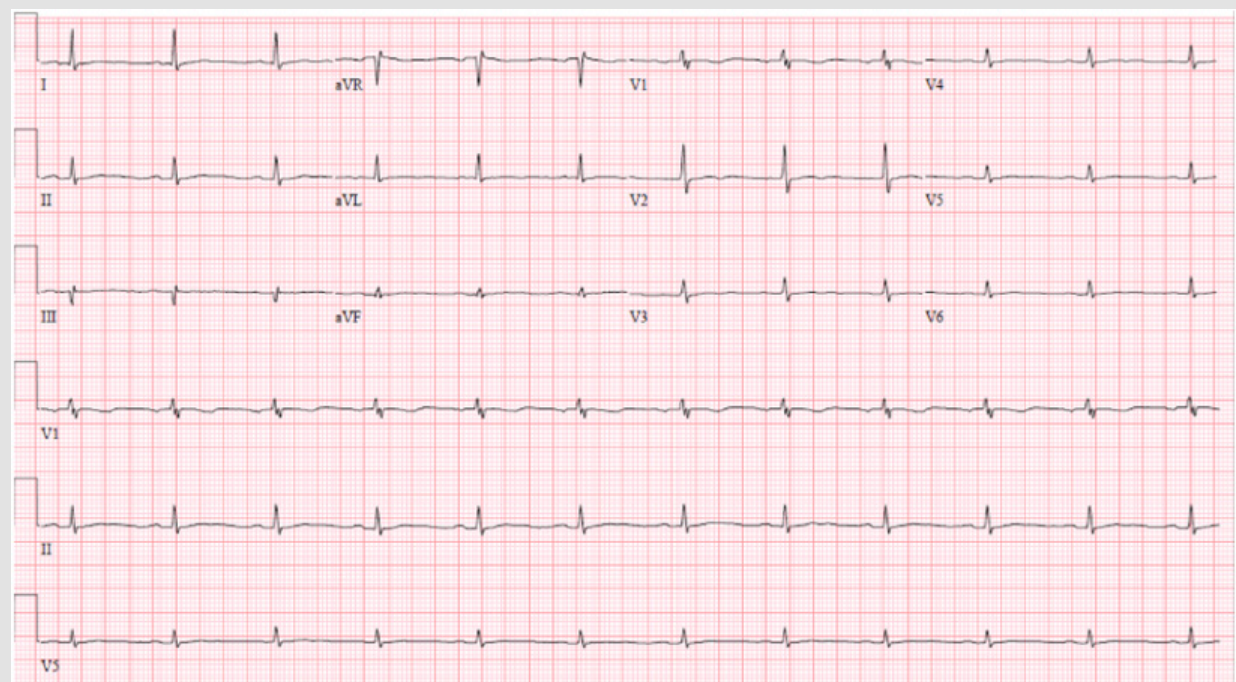

Figure 3: Hospital EKG following Treatment. 


\section{Discussion}

Poison Center consultations for calcium channel blocker (CCB) poisonings are increasing with over 13,000 calls in 2016 [2]. Out of hospital guidelines for CCB poisoning exist but clinical practice has evolved significantly since their publication [3]. This case illustrates the need for rapid, targeted prehospital management of massive CCB poisoning, as these patients are often markedly hemodynamically unstable and critically ill. Multiple doses of calcium chloride were given to the patient both en route and in the ED. This treatment is widely accepted as first line, and administration of calcium has been shown to increase blood pressure in human case series of CCB poisonings [4]. Common dosing of calcium chloride is $1-2 \mathrm{~g}$ IV every 20-30 minutes. EMS protocols should accommodate this higher than normal dosing or allow for deviation in specific cases of large ingestions. Glucagon is also a mainstay in many EMS calcium channel blocker poisoning protocols; however, it has recently fallen out of favor with toxicologists. It is not included in recent CCB management guidelines by The Society of Critical Care Medicine [5]. The reasoning for glucagon's removal is multifactorial, including lack of evidence, increased risk of vomiting with aspiration and significant cost and availability issues. While the final decision on glucagon administration ultimately resides with medical direction, it is clear that glucagon should not supplant or delay other firstline therapies. Our patient did receive glucagon both by EMS and hospital providers, however in retrospect this may have been detrimental considering the patient had an initial secondary complaint of nausea and vomiting.

Crystalloid IV fluid bolus, atropine administration, and vasopressor use are also considered first-line for treating CCB overdose. Norepinephrine and epinephrine are the preferred vasopressors over dopamine. Atropine may not be helpful, especially in higher grade blocks, but the downside of its use is minimal [5]. We were able to utilize both IV and IO access to aggressively bolus the patient with $0.9 \%$ NS. $20 \mathrm{mcg}$ of push dose epinephrine was also used for vascular support during transport. Considering the high-risk and often treatment resistant nature of massive calcium channel blocker ingestion, EMS treatment protocols should include rapid progression through the first-line therapies if hemodynamics do not improve allowing for early vasopressor support. The patient also received HIET and ILE immediately upon arrival to the ED. HIET is widely accepted and considered a first-line therapy in CCB poisoning [5]. High dose insulin is inotropic and also facilitates improved myocyte carbohydrate metabolism. It also counteracts CCB pancreatic islet cell inhibition of insulin release [6]. This was evident in our patient as the ED blood glucose was $350 \mathrm{mg} / \mathrm{dL}$. ILE is also often considered in CCB toxicity with persistent shock, but limited animal and human studies exist to support its use [7]. Consideration was also given to ECMO therapy, however, the patient stabilized during his hospital stay.
This case report demonstrates the complexity of emergent treatment in patients with significant CCB ingestion. EMS protocols must allow for a multifaceted approach with potential for deviation when dosing calcium, vasopressors and insulin. These treatments are especially critical in situations when long transport times are present. Further prehospital research is needed to determine the most efficacious progression through the various treatment modalities used in CCB toxicity.

\section{Conclusion}

We report a case of massive verapamil ingestion with severe bradycardia and treatment resistant shock. EMS treatment included fluid resuscitation, glucagon, multiple doses of calcium chloride, intravenous epinephrine and transdermal pacing. The patient's condition stabilized during the hospital course following HIET, ILE, transvenous pacing and continuous epinephrine infusion. The prehospital treatment of CCB toxicity must be rapid, aggressive and multi-pronged.

\section{Acknowledgment}

No conflict of interest.

\section{Conflict of Interest}

No conflict of interest.

\section{References}

1. Larkin TM, Cohen-Oram AN, Catalano G, Catalano MC (2013) Overdose with levetiracetam: A case report and review of the literature. Journal of Clinical Pharmacy and Therapeutics 38: 68-70.

2. David D Gummin, James B Mowry, Daniel A Spyker, Daniel E Brooks, Michael O Fraser, et al. (2017) 2016 Annual Report of the American Association of Poison Control Centers' National Poison Data System (NPDS): 34 ${ }^{\text {th }}$ Annual Report, Clinical Toxicology 55 (10): 1072-1254.

3. Olson KR, Erdman AR, Woolf AD, Scharman EJ, Christianson G, et al. (2005) American Association of Poison Control Centers: Calcium channel blocker ingestion: An evidence-based consensus guideline for out-of-hospital management. Clin Toxicol (Phila) 43: 797-822.

4. Ramoska EA, Spiller HA, Winter M, Borys D (1993) A one-year evaluation of calcium channel blocker overdoses: Toxicity and treatment. Ann Emerg Med 22: 196-200.

5. St-Onge M, Anseeuw K, Cantrell FL, Gilchrist IC, Hantson P, et al. (2017) Experts Consensus Recommendations for the Management of Calcium Channel Blocker Poisoning in Adults. Critical Care Medicine 45(3): e306-e315.

6. Kristin M Engebretsen, Kathleen M Kaczmarek, Jenifer Morgan, Joel S Holger (2011) High-dose insulin therapy in beta-blocker and calcium channel-blocker poisoning, Clinical Toxicology 49(4): 277-283.

7. Levine M, Hoffman RS, Lavergne V, et al. (2016) Lipid Emulsion Workgroup: Systematic review of the effect of intravenous lipid emulsion therapy for non-local anesthetics toxicity. Clin Toxicol (Phila) 54: 194-221. 


\section{ISSN: 2574-1241}

DOI: $10.26717 /$ BJSTR.2020.25.004226

Casey Patrick. Biomed J Sci \& Tech Res

(C) (P) This work is licensed under Creative

Submission Link: https://biomedres.us/submit-manuscript.php

\begin{tabular}{ll} 
BIOMEDICAL & Assets of Publishing with us \\
RESEARCHES & - Global archiving of articles \\
& - Immediate, unrestricted online access \\
\hline
\end{tabular}

